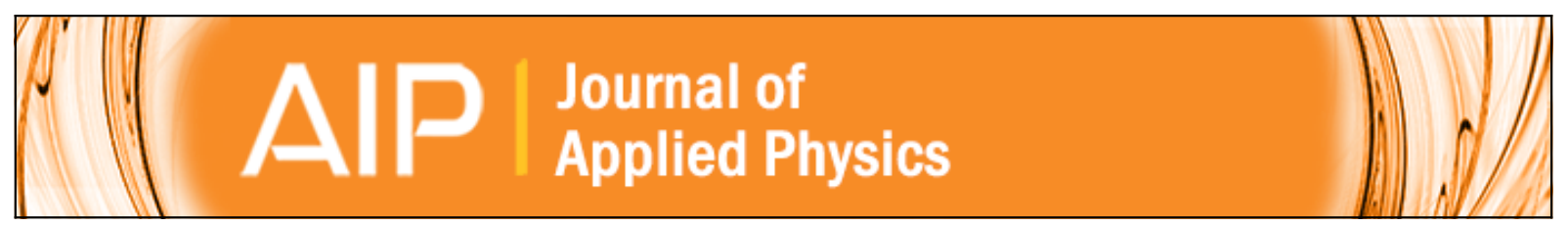

\title{
CdSe/ZnS quantum dot fluorescence spectra shape-based thermometry via neural network reconstruction
}

Troy Munro, Liwang Liu, Christ Glorieux, and Heng Ban

Citation: Journal of Applied Physics 119, 214903 (2016); doi: 10.1063/1.4953223

View online: http://dx.doi.org/10.1063/1.4953223

View Table of Contents: http://scitation.aip.org/content/aip/journal/jap/119/21?ver=pdfcov

Published by the AIP Publishing

\section{Articles you may be interested in}

Fluorescence resonance energy transfer measured by spatial photon migration in CdSe-ZnS quantum dots colloidal systems as a function of concentration

Appl. Phys. Lett. 105, 203108 (2014); 10.1063/1.4902223

Fluorescence quantum efficiency of $\mathrm{CdSe} / \mathrm{ZnS}$ quantum dots embedded in biofluids: $\mathrm{pH}$ dependence J. Appl. Phys. 112, 104704 (2012); 10.1063/1.4767470

Enhancement of the Purcell effect for colloidal CdSe/ZnS quantum dots coupled to silver nanowires by a metallic tip

Appl. Phys. Lett. 100, 253110 (2012); 10.1063/1.4729890

Photon statistics in enhanced fluorescence from a single CdSe/ZnS quantum dot in the vicinity of silver nanoparticles

Appl. Phys. Lett. 95, 193106 (2009); 10.1063/1.3259792

Blinking suppression of colloidal $\mathrm{CdSe} / \mathrm{ZnS}$ quantum dots by coupling to silver nanoprisms

Appl. Phys. Lett. 94, 243108 (2009); 10.1063/1.3154551

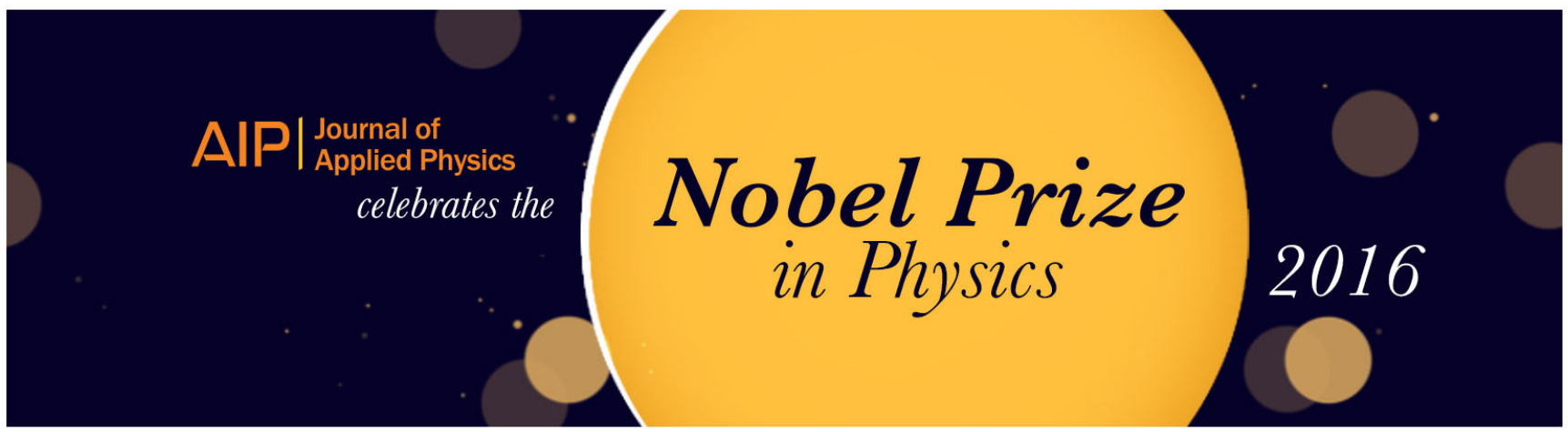




\title{
CdSe/ZnS quantum dot fluorescence spectra shape-based thermometry via neural network reconstruction
}

\author{
Troy Munro, ${ }^{1,2}$ Liwang Liu, ${ }^{2}$ Christ Glorieux, ${ }^{2}$ and Heng Ban ${ }^{1}$ \\ ${ }^{1}$ Multiscale Thermal-Physics Lab, Department of Mechanical and Aerospace Engineering, \\ Utah State University, 4130 Old Main Hill, Logan, Utah 84322, USA \\ ${ }^{2}$ Laboratory of Soft Matter and Biophysics, Department of Physics and Astronomy, KU Leuven, \\ Celestijnenlaan 200D, B-3001 Heverlee, Belgium
}

(Received 20 October 2015; accepted 23 May 2016; published online 6 June 2016)

\begin{abstract}
As a system of interest gets small, due to the influence of the sensor mass and heat leaks through the sensor contacts, thermal characterization by means of contact temperature measurements becomes cumbersome. Non-contact temperature measurement offers a suitable alternative, provided a reliable relationship between the temperature and the detected signal is available. In this work, exploiting the temperature dependence of their fluorescence spectrum, the use of quantum dots as thermomarkers on the surface of a fiber of interest is demonstrated. The performance is assessed of a series of neural networks that use different spectral shape characteristics as inputs (peak-based-peak intensity, peak wavelength; shape-based - integrated intensity, their ratio, full-width half maximum, peak normalized intensity at certain wavelengths, and summation of intensity over several spectral bands) and that yield at their output the fiber temperature in the optically probed area on a spider silk fiber. Starting from neural networks trained on fluorescence spectra acquired in steady state temperature conditions, numerical simulations are performed to assess the quality of the reconstruction of dynamical temperature changes that are photothermally induced by illuminating the fiber with periodically intensitymodulated light. Comparison of the five neural networks investigated to multiple types of curve fits showed that using neural networks trained on a combination of the spectral characteristics improves the accuracy over use of a single independent input, with the greatest accuracy observed for inputs that included both intensity-based measurements (peak intensity) and shape-based measurements (normalized intensity at multiple wavelengths), with an ultimate accuracy of $0.29 \mathrm{~K}$ via numerical simulation based on experimental observations. The implications are that quantum dots can be used as a more stable and accurate fluorescence thermometer for solid materials and that use of neural networks for temperature reconstruction improves the accuracy of the measurement. Published by AIP Publishing. [http://dx.doi.org/10.1063/1.4953223]
\end{abstract}

\section{INTRODUCTION}

Because of the crucial role of temperature in many phenomena, there is a continuous interest in improved temperature measurements in a variety of conditions, especially with non-contact methods. ${ }^{1}$ One avenue of research has been the use of fluorescent dyes as thermometers because of their emission being sensitive to temperature variations. Fluorescent dyes have been used in many instances including labeling structures in cells, ${ }^{2}$ single photon imaging, ${ }^{3}$ and as temperature sensors, relating the individual spectral features such as peak intensity (PI), peak wavelength (PW or PWL), or the ratio of different spectral peaks, ${ }^{4}$ as well as fluorescent lifetime measurements. ${ }^{5}$ Specifically, Rhodamine B dye has been used as a non-contact temperature sensor in microfluidic devices, ${ }^{6,7}$ to probe the surface temperature between two plates, ${ }^{8}$ and as an additive in polydimethylsiloxane (PDMS) to measure the thermal conductivity of carbon nanotubes. ${ }^{9,10}$ However, all of these applications assumed a linear relation between a single spectral feature (usually the peak intensity) and the temperature. In reality, these relations are substantially non-linear, and it is worthwhile to refine them and exploit the information content in multiple features simultaneously. It is therefore important to take into account the fluorescent behaviors additional sensitivity to quenching, dipole interactions,
$\mathrm{pH},{ }^{11}$ salt concentrations, electron coupling, ${ }^{12}$ and to discriminate between those effects and the effect of temperature.

As will be shown in this paper, for the goal of photothermal experiments on fibers (which involve quite time consuming frequency and detection position scans), the stability of organic dyes can be problematic, as photobleaching effects ${ }^{6}$ hamper accurate calibration at set temperatures, and an improved fluorescent probe must be selected. Inorganic compounds, such as quantum dots, are not affected by this, and therefore have the potential for more robust signals. To this end, quantum dots rather than organic fluorescent dyes were selected in the current investigation.

Quantum dots are nanometer-sized semiconductors, whose emission spectra are controlled by their size. While their fluorescence spectrum is also temperature dependent, compared to organic fluorescent dyes, they exhibit improved stability (including after repeated thermal cycling $^{13}$ ), a higher quantum yield, and a slightly longer lifetime. ${ }^{14}$ They also do not contribute significantly to the thermal diffusivity of the material of interest. ${ }^{15}$ so that they have desirable properties for use as a fluorescent thermoprobe. Additionally, on the timescale of photothermal experiments (with the integration time of the spectrometer on the order of ms), the blinking commonly associated with quantum dots (related to the 
lifetime of the fluorophore on the order of tens of ns) is not an issue. ${ }^{16}$ Another incentive to select quantum dots for the current experiment is the earlier demonstration of their successful integration into both silk worm ${ }^{17}$ and spider ${ }^{18}$ silk (although the purpose of this coating was merely for appearances). The thermal properties of spider silk materials have previously been investigated, ${ }^{19}$ and a synthetically produced silk is the substrate used in the current investigation for future thermal characterization by photothermal methods. The typical accuracy of quantum dot fluorescence thermometry is typically on the order of $1-2 \mathrm{~K},{ }^{20}$ but this work seeks to improve upon that temperature accuracy.

Fluorescence thermometry can generally be broken down into two classes of methods: intensity-based and lifetime-based. Lifetime-based relies on pulsed illumination of the fluorophore and detection of the decay time of the excited electrons and emitted photons. ${ }^{21}$ With typical lifetimes on the order of ns, instrumentation and analysis of the signal can be complex and expensive. ${ }^{22}$ Intensity-based methods rely on continuous illumination by the light source and detection by a photodiode, photomultiplier tube (PMT), $\mathrm{CCD}$, or spectrometer (typically). ${ }^{4}$ The benefits of intensitybased methods are reduced complexity and cost, as well as the ease of mapping the acquired temperature signal into the frequency domain for future use during modulated heating experiments. However, they suffer from stability concerns that are not present with lifetime based methods. The proposed work details a data reduction method by neural networks (NNs) to overcome some of these limitations to improve temperature accuracy. These methods could also be applied to other calibration procedures for improved accuracy, such as with molecular tagging thermometry with phosphorescent probes. $^{23}$ However, the current study did not consider phosphorescent thermometry because the typical lifetimes for these probes (on the order of hundreds of $\mu$ s to seconds ${ }^{22}$ ) is not favorable for modulated heating experiments at higher frequencies, although they can have good accuracy $\left(0.8 \mathrm{~K}\right.$ for a point, ${ }^{23} 0.1$ for an interrogation $\left.\operatorname{area}^{24}\right)$.

As mentioned above, in order to further improve the use of quantum dots as temperature sensors, an interesting pathway is to simultaneously use multiple spectral features to extract the temperature. Recently, ${ }^{25}$ the feasibility of using spectral features of organic dyes as inputs for an artificial neural network $(\mathrm{NN})$ and training it to reconstruct the associated temperature has been demonstrated. Neural networks have been used to solve the inverse problem for depth profiling of heat source distribution, ${ }^{26}$ optical penetration via photothermal radiometry, ${ }^{27}$ to relate sea color from satellite imagery to chlorophyll concentrations, ${ }^{28}$ and to determine the thermal diffusivity of a slab of insulation material. ${ }^{29}$ By making use of non-linear functions such as hyperbolic tangent sigmoids, neural network approaches are particularly efficient in dealing with non-linear aspects of the inverse problem. ${ }^{30,31}$ This is an improvement over a simple, standard least squares fit by a sigmoidal function of the spectral feature $^{32}$ required to create the non-linear temperature relationship.

In this work, we verify using a neural network approach for extracting temperature from the fluorescence spectrum of inorganic fluorophores, in the time domain. The neural network is employed to increase the accuracy of the temperature reconstruction compared to a standard linear (as well as exponential, power, smoothing spline, polynomial curve, and polynomial surface) fit. This paper details a photothermal, inorganic fluorescent thermometry accomplished by a time domain temperature reconstruction via neural networks (with inputs to the network based on spectral features of the fluorophore at calibrated temperatures), based on modulated laser heating experiments and simulation.

\section{EXPERIMENTAL SETUP}

\section{A. Equipment description}

The experimental setup consisted of a pump and probe laser system and a sample holder with temperature measurement and control. The fluorescence spectra of Rhodamine B and quantum dots on the sample surface were collected by a microscope objective lens and collimated into the fiber of a USB4000 spectrometer. The optical system that focused the light of the pump laser onto a small spot on the fiber was placed on a position scanning stage (Figure 1). A $532 \mathrm{~nm}$ Coherent Compass CW laser was used to induce the fluorescence at the fiber surface. The laser beam was focused to a spot size of $30 \mu \mathrm{m}$ by means of the top half of a planoconvex lens cut in half. A mechanically chopped laser beam from a $1064 \mathrm{~nm}$ Coherent Vector laser was used to photothermally generate temperature oscillations along the fiber surface. The pump beam diameter on the fiber surface was about $90 \mu \mathrm{m}$. The longer wavelength of the heating laser was selected because it does not induce additional fluorescence in the quantum dots, since the quantum dot absorption is almost negligible at long wavelengths, and the pump wavelength is longer than the one of the fluorescence light. ${ }^{16}$ Besides the IR light that was used for the photothermal excitation, the laser beam of the Coherent Vector laser also contained a weak residual green component. This was reflected and filtered out prior to arriving at the fiber sample. The reflected green light was sent to the spectrometer to provide to the spectrometer a reference signal synchronous with the photothermal excitation. The IR pump light passed through

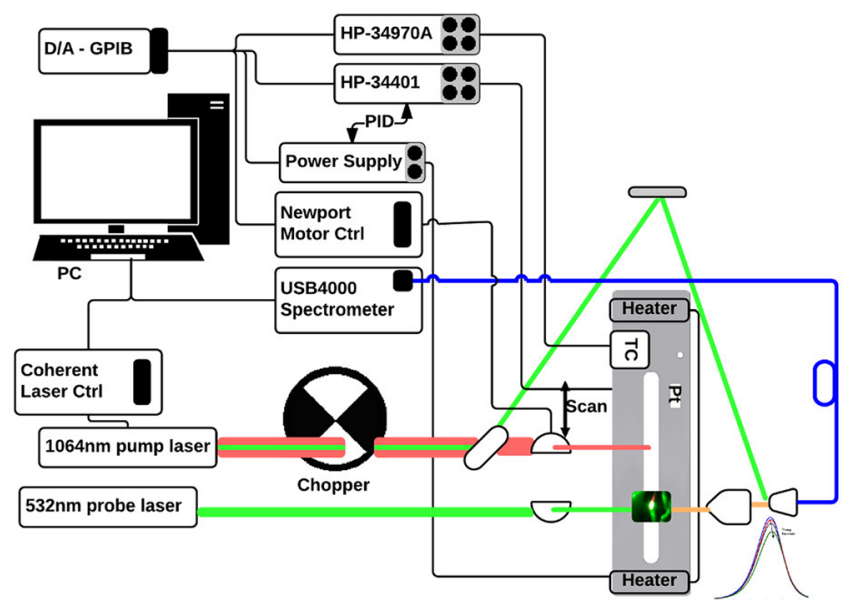

FIG. 1. Experimental setup for fluorescence measurement. 
the bottom half of the split plano-convex lens, which was placed on a linear motor stage with sub-micron resolution, so as to provide accurate axial positioning along the fiber. The half lenses that focused respectively the probe and pump beam to the fiber were placed right on top of each other, in order to have their focal points near to each other, while retaining the possibility to individually scan the focal point of the pump laser beam along the fiber axis by moving the respective half lens along with the beam.

The sample fiber was placed on an aluminum plate, which provided four heating resistors for control of the sample temperature. The temperature of the plate was measured by both an Omega F3141 PT1000 RTD (measured by an HP34401 , with an uncertainty of $0.065 \mathrm{~K}$, based on calibration inside of a furnace used in adiabatic scanning calorimetry ${ }^{33}$ ) and an in-house welded Omega type $\mathrm{T}$ thermocouple (via HP-34970 A, with an uncertainty of $0.1 \mathrm{~K}$, based on a similar calibration) to ensure that the ends of the fiber were near the same temperature, with their location shown in Figure 1. The temperature control of the plate was based on the PT1000 using a PID subroutine in Labview, which controlled the heating resistor voltage supplied by an Agilent E3631A power supply. The sample mount plate was placed inside of a Janis cryostat in order to combine optical access with a vacuum environment. At a pressure of $10^{-5} \mathrm{mTorr}$, the thermal conductivity of the surrounding medium was sufficiently low to allow heat conduction via the air to be neglected in modeling and analyzing the heat transport in and around the fiber. The collection of the emitted fluorescent light was done by a microscope objective $(10 \times, 0.25 \mathrm{NA})$, which was focused onto a fiber optic connected to the Ocean Optics USB4000 spectrometer, after passing through several filters (visible bandpass, short pass, and green notch filters). The green reference signal reflected from the pump laser was also collected in the spectrometer without passing through the filters.

The operation of the experiment was controlled automatically by Labview and began with stabilization of the fiber temperature. Once the temperature variation was under $0.015^{\circ} \mathrm{C}$ for $15 \mathrm{~min}$ (to ensure transients caused by the PID controller could be ignored), the spectrometer collected 250 spectra while keeping track of the temperature as measured by the PT1000 resistance. The means and standard deviations of 1750 spectra between $300 \mathrm{~K}$ and $312 \mathrm{~K}$ were determined, and the individual spectra were stored in order to serve as training data for the neural network.

After the spectral calibration was done, the pump and probe beams were aligned on the fiber, and the position of the pump laser was scanned in steps of $50 \mu \mathrm{m}$ along the fiber in order to map the thermal wave decay versus pump-probe distance between $2000 \mu \mathrm{m}$ and $-2000 \mu \mathrm{m}$. The pump laser was turned on to full power $(1 \mathrm{~W})$ via serial communication to provide sufficient modulated heating of the fiber to produce a photothermal signal, a waiting period of $5 \mathrm{~s}$ occurred to allow a steady state of the DC heating of fiber to be more readily reached so that only the AC variation would be present in the signal, and the spectrometer was set to measure as quickly as possible (after a single measurement from the PT1000 to verify the initial temperature), collecting enough spectra to record 100 periods per pump-probe distance. The temperature of the sample mount could be set at any temperature of interest, allowing performance of frequency and/or position scans, in view of applying the setup in the future to determine the temperature dependence of the thermal diffusivity of the fiber.

\section{B. Fluorophore selection}

Two types of fluorophore-coated fibers were investigated using Rhodamine B and quantum dots as thermoprobes, with the quantum dot coated fiber showing greater stability. The experimental system was first tested on a synthetic spider silk that had Rhodamine B integrated into the fiber during its production, similar to how Rhodamine B had been integrated into an optical fiber for use as a temperature sensor in Ref. 34. The production process ${ }^{35}$ involved coagulation of a fiber in an IPA bath, passing through a methanol/ water bath where it was stretched $1.5 \times$, and then through a water bath, where $65 \mathrm{mg}$ of Rhodamine B was dissolved in $200 \mathrm{ml}$ of distilled water, and stretched again $1.5 \times$. This allowed the Rhodamine B to be integrated throughout the fiber surface, although at an unknown concentration. However, the concentration did not change during the calibration nor the experiment and hence should have little effect on the results. The fiber diameter was about $25 \mu \mathrm{m}$ and thus could be considered to be thermally thin for the used modulation frequencies between $0.5 \mathrm{~Hz}$ and $4 \mathrm{~Hz}$, i.e., the temperature oscillation was rather uniform throughout the fiber, and signal variations were mainly along the fiber axis, with increasing distance from the pump laser spot.

Rhodamine B was initially selected as a thermosensitive probe because it had successfully been used as a temperature sensor in an application for photothermal, shape-based neural network temperature determination, which was aimed at depth profiling of an optical absorbance profile in glycerol. ${ }^{25}$ An important issue encountered with using the Rhodamine B embedded fibers was that the stability of the peak intensity and fluorescent emission spectra was not-consistent over the time needed for a measurement. For this reason, we have started using the more stable Lumidot ${ }^{\mathrm{TM}} 640 \mathrm{~nm}$ peak wavelength, $\mathrm{CdSe} / \mathrm{ZnS}$ quantum dots from Sigma Aldrich. The stabilities over several hours (a typical photothermal experiment at low frequencies and multiple axial positions can take an entire work day) of a synthetic spider silk embedded with Rhodamine B, and the same type of synthetic spider silk coated with the quantum dots, are compared in Figure 2. Quantum dots showed greatly improved stability of the peak intensity. In order to coat the fiber surface with quantum dots, they were first suspended in toluene and then diluted from $5 \mathrm{mg} / \mathrm{ml}$ to $0.5 \mathrm{mg} / \mathrm{ml}$. Next, a drop was placed on the fiber and the toluene was allowed to evaporate, leaving the dots behind the fiber and glass slide. The final concentration on the fiber was unknown, but it was sufficiently high so that the color of the fiber was changed to the color of the dots in solution (red-orange).

Although quantum dots can often experience photodarkening and photobrightening, the illumination of the quantum dot coated sample was for several hours prior to both the 


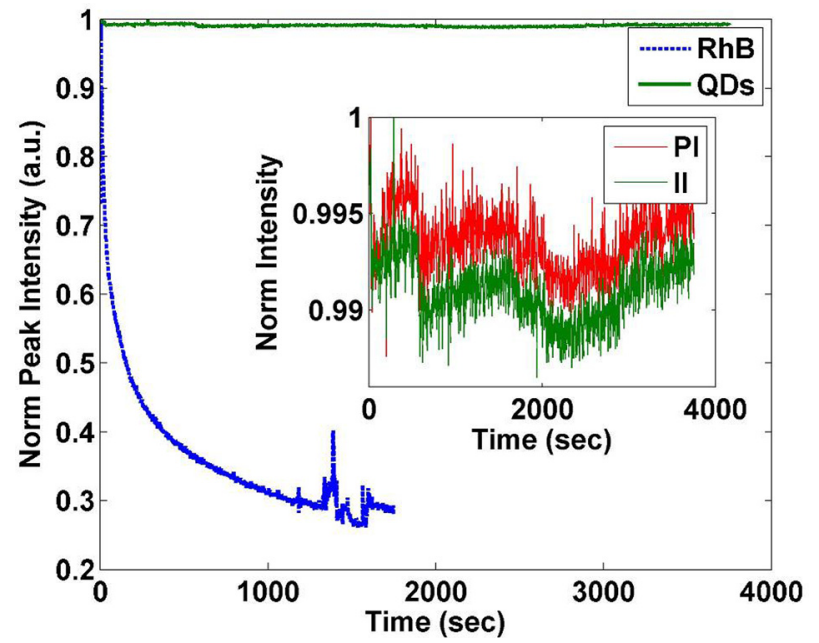

FIG. 2. Comparison of the stability over several hours of the peak intensity of Rhodamine B (blue) and quantum dot coated synthetic spider silk (red). Also, the integrated intensity of the fluorescence peak of the quantum dot coated synthetic spider silk is shown.

calibration and experimental procedures. Hence, the time scales for photodarkening $(3 \mathrm{~s})$ and photobrightening $(>50 \mathrm{~s})^{36}$ are not a concern. Furthermore, the photooxidation that is the mechanism for photodarkening is avoided because the experiment occurs under high vacuum $\left(10^{-5}\right.$ mTorr $) .^{36-38}$ This stability $^{39}$ is also demonstrated in Figure 2, and typical stabilities were observed over three days of separate experiments.

\section{METHODS}

In order to explain the quantum dot fluorescence spectrum-based thermometry, the following sections cover the procedure to train a neural network based on different spectral features to reconstruct the surface temperature in time domain (Sections III A 1 and III A 2) and the numerical experiment (Section III B). This is to determine the viability of this method to accurately determine the dependence of different spectral features on the neural networkreconstructed photothermally induced temperature oscillations based on commonly used spectral features (assuming a linear relation between spectral features and temperature because linear fits are commonly employed in the literature). Further, the selection of a linear relationship for temperature as a function of all spectral features was chosen for simplicity and consistency in development of the numerical model and because the inverse function (spectra feature as a function of temperature) was easily performed.

\section{A. Neural network training and temperature reconstruction}

\section{Temperature behavior of spectra}

Central to the quantum dot fluorescence spectrum shapebased thermometry method is the ability to accurately relate measured spectra to the corresponding fiber surface temperature. In the following, we cover the relation between different spectral features and the DC temperature (without photothermal excitation) and detail the data reduction process to use these spectral features as inputs to train a neural network.

The temperature dependent behavior of the quantum dots spectra and shape-based factors (spectral features) such as peak intensity (PI), integrated intensity (II) ${ }^{40}$ the PI/II ratio $(\mathrm{R}){ }^{41}$ peak wavelength ( $\mathrm{PW}$ or $\mathrm{PWL}^{8}$ ), full-width half maximum (FWHM), ${ }^{8}$ and the summed spectral bands are shown in Figure 3. As expected, $8,25,40,41$ as the temperature increases, the spectral content shifts towards longer wavelengths, broadens, and decreases in intensity (Figure 3(b)). The spectral features mimic this behavior (inset of Figure 3(a)).

The temperature behavior of the fluorescent probe (being established as sufficiently stable) allowed tackling of the inverse problem for extracting the surface temperature from the fluorescence spectrum by means of neural network recognition. The neural network data acquisition process began by combining the 250 calibration spectra from each

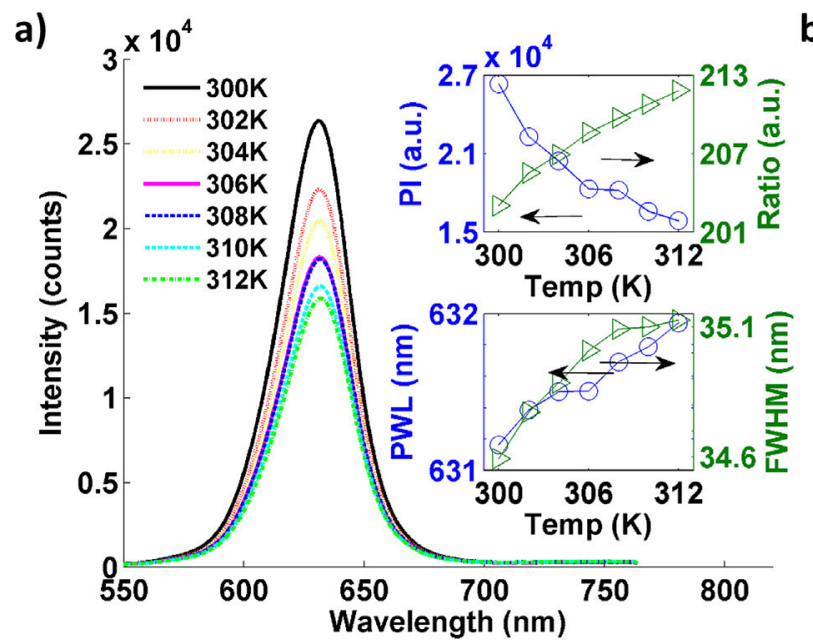

b)

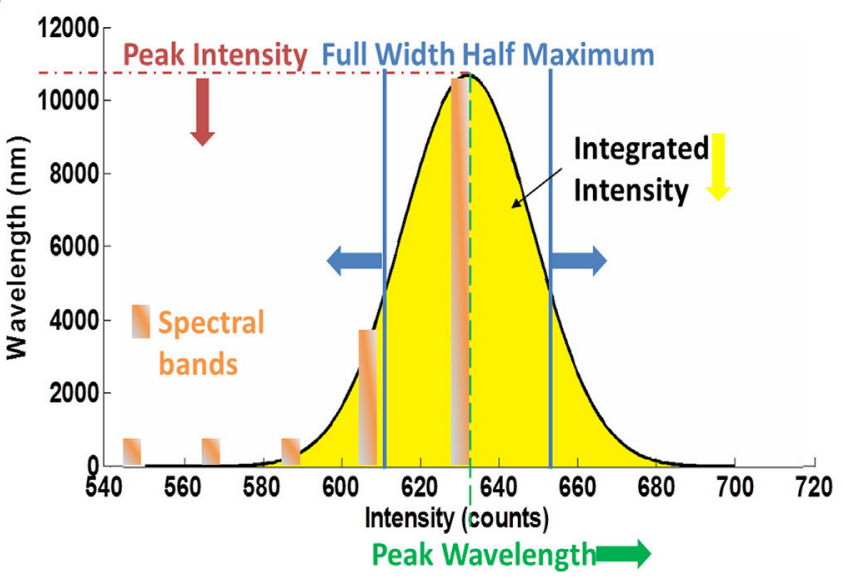

FIG. 3. Experimentally determined temperature behavior of the fluorescent spectra of quantum dots with inset showing the temperature dependence of the PI, Ratio, PWL, and FWHM of the spectra (a). Also, the trend of the spectral features with increasing temperature is schematically illustrated (b). The uncertainty based on a linear fit of the data between $300 \mathrm{~K}$ and $308 \mathrm{~K}$ and precision of each parameter are: PI 1.1 K, PWL 1.4, II 2.0 K, Ratio, 1.2 K, FWHM 1.5 K, and normalized intensity at a single wavelength $1.8 \mathrm{~K}$. Neural networks trained on these different features are expected to be able to increase the accuracy of temperature reconstruction from spectral data. A summary of results with other fits based solely on the RMS error of the fit to the calibrated data is presented in Section IV B. 
calibration temperature into a single file and performing a moving, rectangular-windowed average (10 data points), where the intensity of the counted intensity was averaged over 10 wavelengths, in order to reduce the noise and determine the features of interest (peak intensity, integrated intensity, peak wavelength, ratio of peak to integrated intensity, FWHM, peak normalized intensities, and summed bands) more accurately and more easily.

A 3rd order polynomial was fit to the smoothed spectra, the derivative was taken, and the peak intensity and peak wavelength were found at the maximum of the fit curve. The selection of the polynomial order was motivated by the desire to have a numerical stable derivative and to be able to be a more general fit because of the different shaped-spectra of the Rhodamine B and quantum dot spectra. The integrated intensity was then taken as the sum of the intensity values under the curve of the smoothed spectra. Also, the ratio between the peak intensity and the integrated intensity of the spectra was calculated. The FWHM of the spectral peak was determined as well. The spectra were normalized to their peak value and the "normalized intensity" values at 40 evenly spaced wavelengths from $536 \mathrm{~nm}$ to $764 \mathrm{~nm}$ were used as inputs for the neural networks. Finally, five bands of 25 wavelength measurements ( $\sim 5 \mathrm{~nm}$ wide) were summed, and these five "sum bands" would provide the inputs for one of the neural networks.

\section{Neural network training}

The different spectral features were used as inputs to create five different, simple neural networks containing 2 hidden nodes, with varying inputs based on the different spectral features just described and summarized below:

1. PI, II, PWL with 2 hidden nodes.

2. PI, II with 2 hidden nodes.

3. PI, II, R, FWHM, PWL with 2 hidden nodes.

4. PI, 40 Normalized Intensities with 2 hidden nodes.

5. Sum Bands with 2 hidden nodes.

These were selected to represent traditional methods for temperature correlations (peak intensity and wavelength), shapebased methods (FWHM and integrated intensity), and combinations of both methods (normalized intensity and sum bands).

The actual training of the neural network used $80 \%$ of the calibrated spectra for training the network and 20\% for testing how well the output matches expectations on new data. Each spectrum was randomly assigned to either group, for cross-validation. The weights of the different nodes (Figure 4(a), with the results of the training presented in Figure 4(b)) were varied at each iteration until the RMS error of the NN output temperature to the expected temperature (from the PT1000 measurement) was sufficiently small, with the variation of the weights of the nodes in each layer being guided by a Levenberg-Marquardt algorithm. The weights of the neural networks were stored and then applied to each measured spectra, in order to determine the modulated temperature of the fiber during photothermal heating.

Figure 4(b) shows that over the temperature range of interest, 300-312 K, there is a satisfactory correlation between the temperature values extracted by the $\mathrm{NN}$ from the spectra
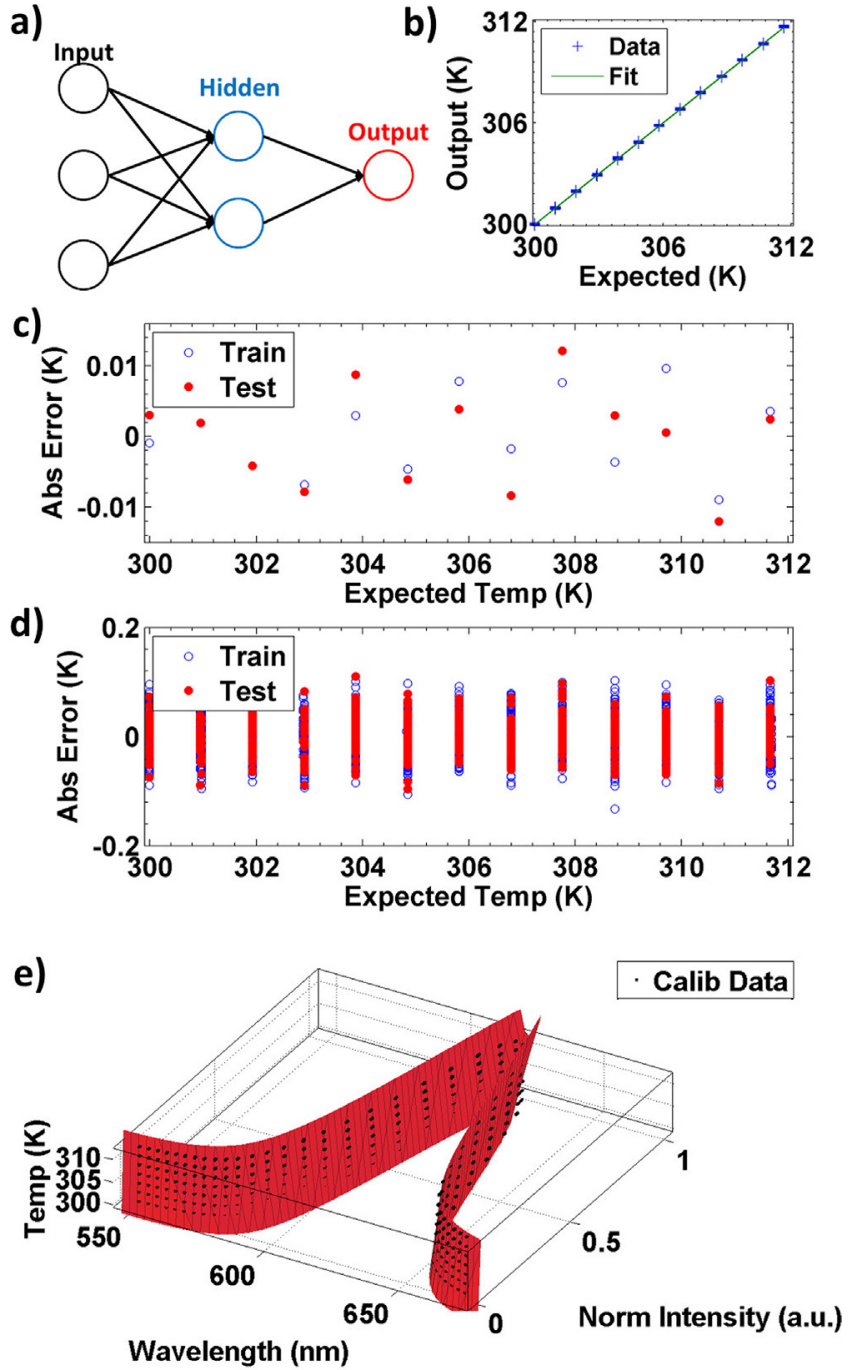

FIG. 4. Typical NN architecture (a), correlation between expected and extracted temperature values (b), and averaged (c) and raw (d) RMS error values versus temperature from the training with the results of training spectra in blue circles and the results of test spectra in red dots, simulated data based on experimental uncertainties. Surface fit to calibrated temperature data as a function of normalized intensity and wavelength (e) as a curve-fit comparison to the $\mathrm{NN}$, with RMS error of $3.5 \mathrm{~K}$.

on one hand, and the actual temperatures on the other hand. Figure 4(c) shows that the average reconstruction error for the training and test data is less than $10 \mathrm{mK}$, with a spread similar on the order of $100 \mathrm{mK}$ (Figure 4(d)) for the normalized intensity-based NN. To provide a valid comparison, a 2D surface fit was performed on the calibrated data where temperature was given as a function of wavelength and peak normalized intensity. Each parameter was fit with a 3rd order polynomial, with the resulting RMS error of the fit being 3.5 K. The results of that fit are given in Figure 4(e), demonstrating the improvement through use of the neural network. Further analysis of the improvement of temperature reconstruction via NN is detailed in Section IV B.

\section{B. Numerical experiment for $\mathrm{NN}$ accuracy}

As mentioned above, the future application of interest in this work is the use of photothermal excitation and fluorescencebased thermometry to extract the thermal diffusivity along the 
axis of a fiber from the axial pump-probe distance dependence of the photothermally induced temperature oscillation amplitude and phase delay with respect to the intensity modulation. In order to determine which neural network provides the best temperature reconstruction, a numerical simulation of the quantum dot spectra and their variation due to temperature was created, with the experimental to simulation comparison given in Figure 5.

The time dependence of the detection temperature was simulated (see further), and noise was added to it to produce simulated spectra. Additional noise was added to the spectra, based on the signal-to-noise ratio of the spectrometer. The simulation took advantage of the almost Gaussian-shape of the quantum dot fluorescence spectra. Based on the experimental observations in Figure 3, the different spectral features were assumed to be linearly related to temperature
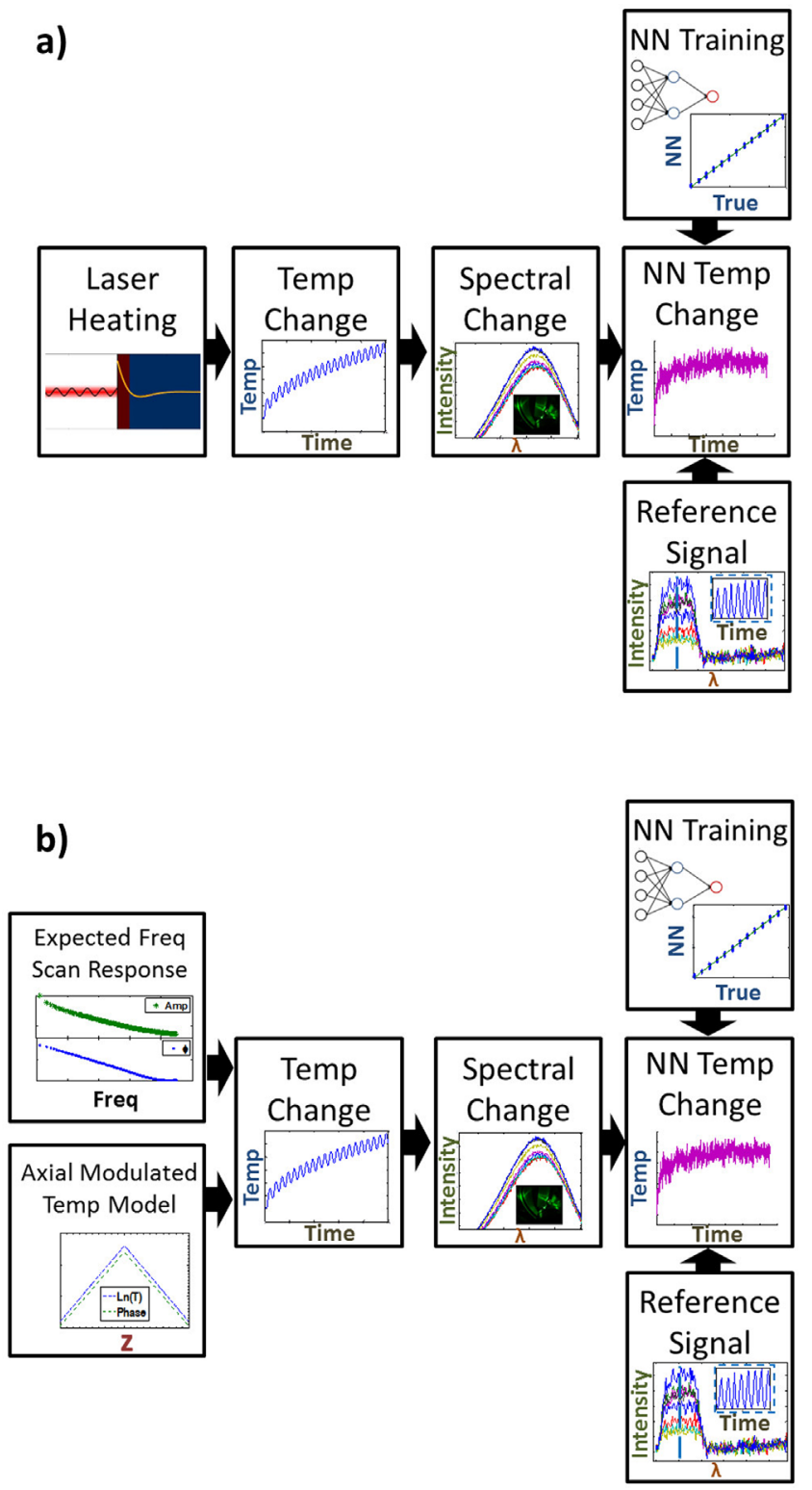

FIG. 5. Schematic illustration of the experimental procedure for calculating the thermal diffusivity of a fiber by determining the dependence on the photothermal signal amplitude and phase as a function of the laser intensity modulation frequency and of the pump-probe distance along the fiber (a) and numerical simulation model (b).
$\left(A=P_{P I, 0}+S_{P I} T\right)$ over the linear region of the temperature range of interest $(300 \mathrm{~K}-308 \mathrm{~K})$. Although the experimental data (temperature as a function of spectral feature) was better fit with a second order polynomial (Figure 8), the assumption of a linear relation was chosen because the inversion of the relationship (spectral feature as a function of temperature) is easily performed on linear relationships. Additionally, it was chosen because it is a common practice in the literature and because the choice of fit does not matter in the comparison to the neural network results as long as the simulated data is analyzed by the same fit that was originally assumed. The resulting temperature determined by the different neural networks could then be compared to the actually modulated temperature that was used to create the spectra.

The model of Salazar et al. ${ }^{42}$ for an infinitesimally narrow laser line heating source was used to represent the complex temperature field of a cylinder for a given periodic intensity modulation, in vacuum, with linearized radiation, and the fiber radius much smaller than the diffusion length of the thermal wave, Eqs. (1) and (2). For the sake of simplicity, the fluorescent spot was assumed to be a point

$$
\begin{gathered}
\tilde{T}_{s}(a, z) \approx \frac{P_{0}}{2 \pi k_{s} q_{s}^{\prime}} e^{-q_{s}^{\prime}|z|}, \\
q^{\prime}=\sqrt{\frac{i \omega}{\alpha_{s}}+\frac{2 h}{k_{s} a}} .
\end{gathered}
$$

The model predicts a linear dependence of the phase delay and of the logarithm of the amplitude on the pumpprobe distance, Eqs. (3) and (4)

$$
\begin{gathered}
z_{\text {mag }}=\exp \left(m_{\text {mag }}|z|+b_{\text {mag }}\right), \\
z_{\text {phase }}=m_{\text {phase }}|z|+b_{\text {phase }} .
\end{gathered}
$$

The slope is determined by the modulation frequency and the thermal diffusivity of the fiber, Eq. (5)

$$
m_{\text {phase }} \times m_{\text {mag }}=-\pi f \alpha_{s}^{-1} .
$$

To mimic the time domain behavior of the experiment, the complex temperature (modelled as an AC signal, Eq. (6)) was superimposed onto a gradual DC temperature rise (Eq. (7)), with a power law shape with exponent $1 / 2$

$$
\begin{gathered}
T_{A C}(\text { or } f, t)=\frac{\text { freq }_{\text {mag }}}{\text { freq }_{\text {mag }, \text { max }}} \frac{z_{\text {mag }}}{z_{\text {mag, } \text { max }}} \times \cos \left[2 / \text { pif }\left(t-t_{0}\right)\right. \\
\left.\quad-z_{\text {phase }}-\text { freq }_{\text {phase }}\right], \\
T_{D C}(\text { or } f, t)=\left[T_{\text {end }}-T_{0}\right]\left(\frac{t-t_{0}}{t_{\text {end }}}\right)^{n}+T_{0} .
\end{gathered}
$$

The time domain temperature evolutions were then used to create Gaussian-shaped fluorescence spectra at different times, Eq. (8), using the assumed linear dependencies of the spectral features $\left(A_{P I, 0}, S_{P I}\right.$, etc.) on temperature from calibration experiments, with the inputs to the model given in Table I. A reference signal, synchronous with the intensity oscillation underlying the simulated temperature evolution, was used to create a trapezoidal curve between $526 \mathrm{~nm}$ and 
TABLE I. Inputs and uncertainties used for the simulation based on calibration experiment from Figure 3.

\begin{tabular}{lcc}
\hline \hline Simulation parameters & Nominal values & Uncertainty \\
\hline Wavelength resolution & $520-770 \mathrm{~nm}$ & $0.19 \mathrm{~nm}$ \\
PT1000 Temp. & $300-312 \mathrm{~K}$ & $0.05 \mathrm{~K}$ \\
Time spacing & $0.001 \mathrm{~s}$ & $0.000727 \mathrm{~s}$ \\
Spectrometer noise & 0 counts & 217 counts \\
$A_{P I, 0}$ & 329356 counts & 42.58 counts \\
$B_{P W L, 0}$ & $613.51 \mathrm{~nm}$ & $0.123 \mathrm{~nm}$ \\
$C_{F W H M, 0}$ & $16.153 \mathrm{~nm}$ & $0.00032 \mathrm{~nm}$ \\
$D_{0}$ & 1500 counts & 50 counts \\
$S_{P I}$ & -1014 counts/K & 1.391 counts $/ \mathrm{K}$ \\
$S_{P W L}$ & $0.059 \mathrm{~nm} / \mathrm{K}$ & $0.000404 \mathrm{~nm} / \mathrm{K}$ \\
$S_{F W H M}$ & $0.0616 \mathrm{~nm} / \mathrm{K}$ & $0.000105 \mathrm{~nm} / \mathrm{K}$ \\
$S_{D}$ & 0 counts/K & 0 counts $/ \mathrm{K}$ \\
Motor position & $0.5 \mathrm{~mm}$ & $0.0011 \mathrm{~mm}$ \\
$m_{\text {phase }}$ & $2000 \mathrm{rad} / \mathrm{m}$ & \\
$m_{\text {mag }}$ & $6001 / \mathrm{m}$ & \\
$\alpha_{s}$ & $0.13 \mathrm{~mm} / \mathrm{s}$ & \\
$P_{0}$ & $1000 \mathrm{a} / \mathrm{u}$. & $0.05 \mathrm{~Hz}$ \\
$k_{s}$ & $0.24 \mathrm{~W} / \mathrm{mK}$ & \\
h & $6 \mathrm{~W} / \mathrm{m}^{2} \mathrm{~K}$ & $0.5 \mathrm{~Hz}$ \\
Frequency & & \\
\hline \hline
\end{tabular}

$535 \mathrm{~nm}$, thus simulating the green light directed into the spectrometer.

$$
\begin{aligned}
\operatorname{Spec}[\lambda, T(z, t)]= & \left(A_{P I, 0}+S_{P I} T\right) \\
& \times \exp \left[\frac{-4 \ln (2)\left(\lambda-\left(B_{P W L, 0}+S_{P W L} T\right)\right)^{2}}{\left(C_{F W H M, 0}+S_{F W H M} T\right)^{2}}\right] .
\end{aligned}
$$

To fully mimic the experiment, this simulation also created calibration files of spectra at fixed temperatures to train the neural network and then created spectra from a modulated temperature at a fixed frequency and axial position, with similar uncertainties to those seen during the experiment, also presented in Table I.

\section{RESULTS}

\section{A. NN accuracy via simulation compared to experiment}

An example of experimentally determined temperature evolutions using the five NNs investigated is shown in Figure 6 for an axial pump-probe spacing of $1 \mathrm{~mm}$ and at an initial temperature near the lower limit of the calibration range $(302 \mathrm{~K})$. All evolutions show the expected DC temperature rise and modulated temperature. However, there is a significant variation in the reconstructed temperature by the different networks, with the normalized intensity $\mathrm{NN}$ having the least variation (Figure 6, inset) and the NN with the most spectral features (PI, II, Ratio, FWHM, PWL) having the most variation. The data presented in the inset of Figure 6 is meant to compare the magnitude of the $\mathrm{AC}$ variation of the different neural networks by normalizing them to their final steady state value, since there is a significant DC offset to NNs \#4 and \#5.

The discrepancy between the different NN reconstructions is postulated to be due to the nature of the inputs to the

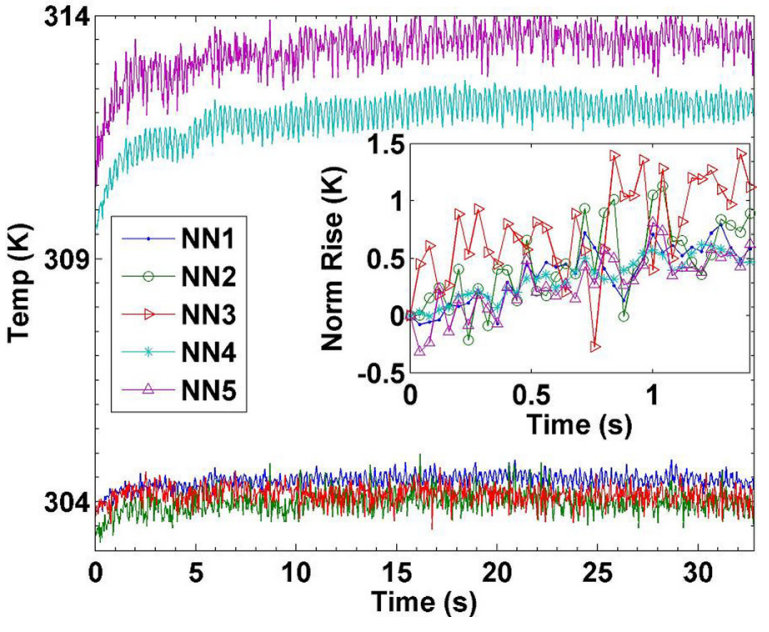

FIG. 6. Neural network reconstructed temperature evolutions for five types of neural network inputs investigated based on experimental data. The inset shows the normalized temperature evolutions. Because of the variation caused by the different neural networks, a numerical simulation was developed to determine which type of inputs provides the greatest accuracy of the reconstructed temperature.

neural networks. NNs \#1-\#3 rely mainly on intensity-based features of the spectra (PI, II, R), while NNs \#4 and \#5 rely on a mix of both intensity-based and shape-based features in the reconstruction of the temperature, which have different accuracies. ${ }^{43}$ However, the ultimate reason for up to a $10 \mathrm{~K}$ difference is unclear and was the incentive to perform this analysis on simulated data, for which the reconstructed data can be unambiguously evaluated by comparison with the known temperature evolution underlying the simulation. Comparison of experimental temperature reconstructions for absolute error under modulated heating is not feasible because the actual temperature of the fiber is unknown during the experiment. As will be shown later, the numerically simulated uncertainty of $1.02 \mathrm{~K}$ via simulation compared to $1.1 \mathrm{~K}$ from experimental linear fit of peak intensity (in the linear region of the temperature range $300 \mathrm{~K}-308 \mathrm{~K}$ ) during the calibration process was sufficiently similar that the uncertainty results of the numerical simulation could be comparable to uncertainty results of the experiment.

The numerical simulation was based on the nominal values of uncertainty on the different spectral features and other system parameters (Table I). The calibration spectra were created based on the magnitude of the neural network parameters observed during the experiment as shown in Figure 3. The neural networks were then trained on those data. Next, the simulation assumed a temperature evolution as described in Section III B. For each temperature, a spectrum was generated with the corresponding characteristics (FWHM, peak intensity, peak wavelength) with noise superimposed onto the created spectra. Finally, the trained neural networks were then used to determine the temperature evolution from the generated spectra. The reconstructed temperature evolutions were compared with the original one, in order to determine which provides the greatest accuracy.

The RMS error on the temperature evolution extracted by the different NNs is included in the legend in Figure 7. The most accurate NN reconstruction was based on the 


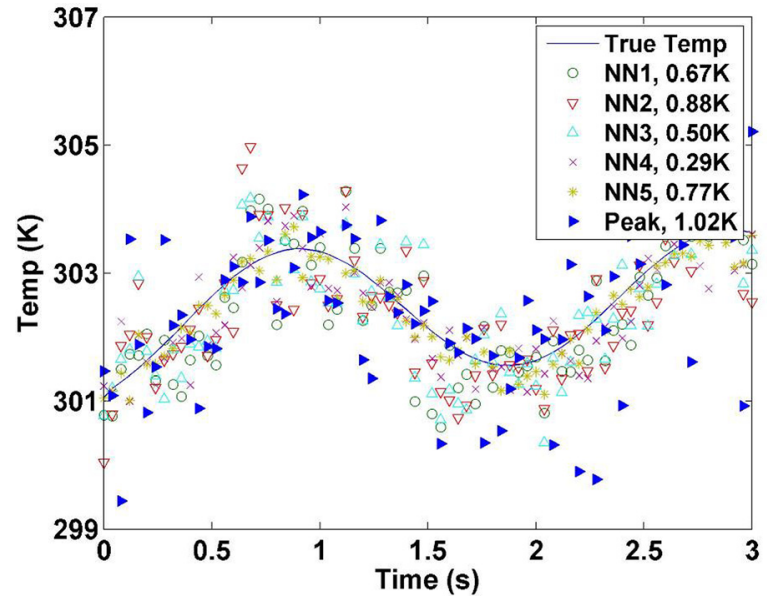

FIG. 7. Reconstructed temperature signals by 5 different neural networks (hollow symbols) and reconstruction by a linear fit to peak intensity (filled symbols), compared to the original temperature (full line), with the RMS error of each (in Kelvin) in the legend. The normalized intensity based neural network matches the input temperature most accurately in the simulation.

normalized intensities with an RMS error lower than that traditionally observed in fluorescent dyes (Ref. 20). The discrepancy observed in the experimental temperature can be attributed to a large DC heating during the initial $5 \mathrm{~s}$ when the pump laser was on but the spectrometer was not collecting data. However, this discrepancy was not observed in the simulated temperature

$$
R M S_{\text {error }(K)}=\sqrt{\sum_{1}^{N}\left(T_{N N}-T_{T r u e}\right)^{2} / N} .
$$

Furthermore, the numerical simulation provides an excellent vehicle to determine the sensitivity of the method to different experimental errors. The inputs of Eq. (8) into the simulation were fixed at their nominal value, except for one, in which the bias and precision uncertainty was $1 \%$ of the nominal value. The numerical experiment was repeated for each input for a fixed axial position and frequency, which then fixed the decay and phase delay terms Eq. (6) as a constant. Calibration spectra and modulated spectra at the lowest temperature of the calibration range were created. The neural network calibration and application was applied to the simulated, modulated spectra, and the final variation from the true modulated temperature compared to the neural network determined temperature was determined. Table II presents the RMS (Eq. (9)) error in Kelvin of the reconstructed temperature, resulting from a $1 \%$ uncertainty in the investigated variables. Apparently, the FWHM based $\mathrm{NN}$ is the most sensitive to deviations. This can be explained by the rather low sensitivity of the FWHM to temperature (about $0.18 \% / \mathrm{K}$ ) and the limited wavelength resolution of the spectrometer $(\approx 0.2 \mathrm{~nm})$.

\section{B. Justification for NN use compared to curve-fit}

Since the objective of this research is to demonstrate the improved temperature resolution via a neural network, this section will compare the results from both experimental data
TABLE II. RMS error (K) of spectral inputs at $1 \%$ uncertainty of each input, with the FWHM being the most sensitive to uncertainty.

\begin{tabular}{lccccc}
\hline \hline & NN\#1 & NN\#2 & NN\#3 & NN\#4 & NN\#5 \\
\hline Z position & 0.14 & 0.48 & 0.47 & 0.16 & 0.10 \\
Wavelength & 0.11 & 0.11 & 0.11 & 0.08 & 0.10 \\
PT1000 Temp. & 1.61 & 1.69 & 4.22 & 1.60 & 1.60 \\
Spectrometer noise & 0.09 & 0.09 & 0.09 & 0.09 & 0.10 \\
Slope of PWL & 0.10 & 0.10 & 0.11 & 0.10 & 0.10 \\
Intercept of PWL & 0.10 & 0.10 & 0.10 & 0.10 & 0.19 \\
Slope of PI & 0.12 & 0.12 & 0.09 & 0.10 & 0.10 \\
Intercept of PI & 0.12 & 0.12 & 0.09 & 0.10 & 0.10 \\
Slope of FWHM & 0.14 & 0.48 & 0.47 & 0.16 & 0.10 \\
Intercept of FWHM & 8.88 & 4.70 & 10.47 & 37.47 & 34.57 \\
Modulation freq. & 0.14 & 0.49 & 0.50 & 0.17 & 0.10 \\
Slope of baseline & 0.14 & 0.48 & 0.47 & 0.16 & 0.10 \\
Intercept of baseline & 0.14 & 0.48 & 0.47 & 0.16 & 0.10 \\
\hline \hline
\end{tabular}

and simulated data to demonstrate this point. The results of multiple types of curve fits (linear, polynomial, exponential, smoothed spline, interpolated, 2D surface fits, etc.) compared to neural networks are shown in Table III. Atypical neural networks were constructed based on a single input to compare the results of single parameter fits of the same spectral feature. Furthermore, the results based on experimental fits can only present the error associated with the fitting of the curve fit model curve or error of the neural network reconstructed temperature compared to the calibrated spectra at references temperatures. Results from the actual operation of the experiment during modulated heating were not investigated because the temperature of the fiber was not known. To consider situations where the fiber temperature is known, the simulation described in Section III B was performed, and the RMS error of the difference between the reconstructed and simulated temperature was presented. Only linear fits were considered for the reconstruction because the simulation explicitly used a linear relationship between the temperature and spectral features. The results of the simulated curve fit relationships are given in Figure 8 and show the high degree of linearity of the data $\left(\mathrm{R}^{2} \approx 0.99\right)$, while the differences in temperature reconstruction for a linear fit to the peak intensity and neural networks is given in Figure 7.

Among the benefits of the neural network is the ability to combine data from multiple parameters into a single output. This is possible and easily visualized for twodimensional surface fits, but as the number of parameters increase, this method becomes more difficult and cumbersome. Two examples are given with the curve fits similar to NN \#2 (PI, II) and NN \#4 (PI, 40 Norm). With two parameters (PI and II), the 3D surface of temperature is easily created, and the resulting error is similar to that of the neural network. With 41 parameters, the improvement in error is by a factor of almost $\sqrt{40}$, and the 3D surface of temperatures must be constructed as a function of wavelength and normalized intensity at that wavelength rather than letting the intensity at each wavelength be a separate parameter. By doing this, the ability to reduce the error by a factor of $\sqrt{N}$ is eliminated since there is no weighting function to consider all wavelengths of interest and the normalized intensity at only 
TABLE III. Comparison of curve fits to neural network reconstruction for both experiment and simulation.

\begin{tabular}{|c|c|c|c|c|c|c|c|c|c|c|c|c|}
\hline \multicolumn{13}{|c|}{ Experiment } \\
\hline \multirow[b]{2}{*}{ Parameter } & \multicolumn{6}{|c|}{ Based only on fit } & \multicolumn{6}{|c|}{ Absolute test RMSE } \\
\hline & Lin & Exp & Power & Spline & 2nd Poly & Surf & 1 st NN & NN \#1 & NN \#2 & NN \#3 & NN \#4 & NN \#5 \\
\hline PI & 1.4 & 1.4 & 1.1 & 1.1 & 0.67 & $\ldots$ & 0.24 & $\ldots$ & $\ldots$ & $\ldots$ & $\ldots$ & $\ldots$ \\
\hline PWL & 0.83 & 0.82 & 0.82 & 1.3 & 0.9 & $\ldots$ & 0.088 & $\ldots$ & $\ldots$ & $\ldots$ & $\ldots$ & $\cdots$ \\
\hline FWHM & 1.5 & 1.5 & 1.5 & 0.9 & 1.1 & $\ldots$ & 0.68 & $\ldots$ & $\ldots$ & $\ldots$ & $\ldots$ & $\ldots$ \\
\hline II & 1.4 & 1.4 & 1.1 & 1.3 & 0.72 & $\ldots$ & 0.099 & $\ldots$ & $\ldots$ & $\ldots$ & $\ldots$ & $\ldots$ \\
\hline Ratio & 0.74 & 0.71 & 0.74 & 0.15 & 0.16 & $\ldots$ & 0.19 & $\ldots$ & $\ldots$ & $\ldots$ & $\ldots$ & $\ldots$ \\
\hline Norm Intensity @1 $\lambda$ & 0.85 & 0.85 & 0.85 & 1.3 & 0.93 & $\ldots$ & 0.28 & $\ldots$ & $\ldots$ & $\ldots$ & $\ldots$ & - \\
\hline PI, II, PWL & $\ldots$ & $\ldots$ & $\ldots$ & $\ldots$ & $\ldots \ldots$ & $\cdots$ & $\ldots$ & 0.05 & $\ldots$ & $\cdots$ & $\ldots$ & $\cdots$ \\
\hline PI, II & $\ldots$ & $\ldots$ & $\ldots$ & $\ldots$ & $\ldots$ & 1.59 & $\ldots$ & $\ldots$ & 0.08 & $\ldots$ & $\ldots$ & $\ldots$ \\
\hline PI, II, R, FWHM, PWL & $\ldots$ & $\ldots$ & $\ldots$ & $\ldots \ldots$ & $\ldots$ & $\ldots$ & $\ldots$ & $\ldots$ & $\ldots$ & 0.07 & $\ldots$ & $\ldots$ \\
\hline PI, 40 norm & $\ldots$ & $\ldots$ & $\ldots$ & $\ldots$ & $\ldots$ & 3.5, Fig. 4(e) & $\ldots$ & $\ldots$ & $\ldots$ & $\ldots$ & 0.016 & $\ldots$ \\
\hline Sum bands & $\ldots$ & $\ldots$ & $\ldots$ & $\ldots$ & $\ldots$ & 2.7 & $\ldots$ & $\ldots$ & $\ldots$ & $\ldots$ & $\ldots$ & 0.084 \\
\hline
\end{tabular}

RMSE from simulated temperature

\begin{tabular}{|c|c|c|c|c|c|c|c|c|c|c|c|c|}
\hline \multirow[b]{2}{*}{ Parameter } & \\
\hline & Lin & Exp & Power & Spline & 2nd Poly & Surf & 1 st NN & NN \#1 & NN \#2 & NN \#3 & NN \#4 & $\mathrm{NN} \# 5$ \\
\hline PI & 1.02 & $\ldots$ & $\ldots$ & $\ldots$ & $\ldots$ & $\ldots$ & $\ldots$ & $\ldots$ & $\ldots$ & $\ldots$ & $\ldots$ & \\
\hline PWL & 1.03 & $\cdots$ & $\cdots$ & $\cdots$ & $\cdots$ & $\cdots$ & $\cdots$ & $\cdots$ & $\cdots$ & $\cdots$ & $\cdots$ & \\
\hline FWHM & 0.69 & $\ldots$ & $\ldots$ & $\ldots$ & $\ldots$ & $\ldots$ & $\ldots$ & $\ldots$ & $\ldots$ & $\ldots$ & $\ldots$ & \\
\hline II & 2.56 & $\ldots$ & $\ldots$ & $\ldots$ & $\ldots$ & $\ldots$ & $\ldots$ & $\ldots$ & $\ldots$ & $\ldots$ & $\ldots$ & \\
\hline Ratio & 2.56 & $\cdots$ & $\cdots$ & $\cdots$ & $\cdots$ & $\cdots$ & $\cdots$ & $\cdots$ & $\cdots$ & $\cdots$ & $\cdots$ & \\
\hline 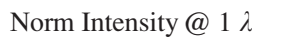 & 2.06 & $\ldots$ & $\ldots$ & $\ldots$ & $\ldots$ & $\cdots$ & $\ldots$ & $\ldots$ & $\ldots$ & $\ldots$ & $\ldots$ & \\
\hline PI, II, PWL & $\ldots$ & $\cdots$ & $\cdots$ & $\cdots$ & $\cdots$ & $\cdots$ & $\cdots$ & 0.67 & $\cdots$ & $\cdots$ & $\ldots$ & $\cdots$ \\
\hline PI, II & $\ldots$ & $\ldots$ & $\ldots$ & $\ldots$ & $\ldots$ & 0.9 & $\ldots$ & $\ldots$ & 0.88 & $\ldots$ & $\ldots$ & $\ldots$ \\
\hline PI, II, R, FWHM, PWL & $\ldots$ & $\ldots$ & $\ldots$ & $\ldots$ & $\ldots$ & $\ldots$ & $\ldots$ & $\ldots$ & $\cdots$ & 0.5 & $\ldots$ & $\ldots$ \\
\hline PI, 40 norm & $\cdots$ & $\cdots$ & $\cdots$ & $\cdots$ & $\cdots$ & 2.84 & $\cdots$ & $\cdots$ & $\cdots$ & $\cdots$ & 0.29 & $\cdots$ \\
\hline Sum bands & $\cdots$ & $\cdots$ & $\cdots$ & $\cdots$ & $\cdots$ & 0.83 & $\ldots$ & $\ldots$ & $\cdots$ & $\cdots$ & $\cdots$ & 0.77 \\
\hline
\end{tabular}

one value can be considered for determination of the temperature on the surface fit.

Finally, the experimental results presented in Table III show that the curve and surface fits are out-performed by their equivalent neural network reconstructions, with the exception of the smoothing spline and 2nd order polynomial
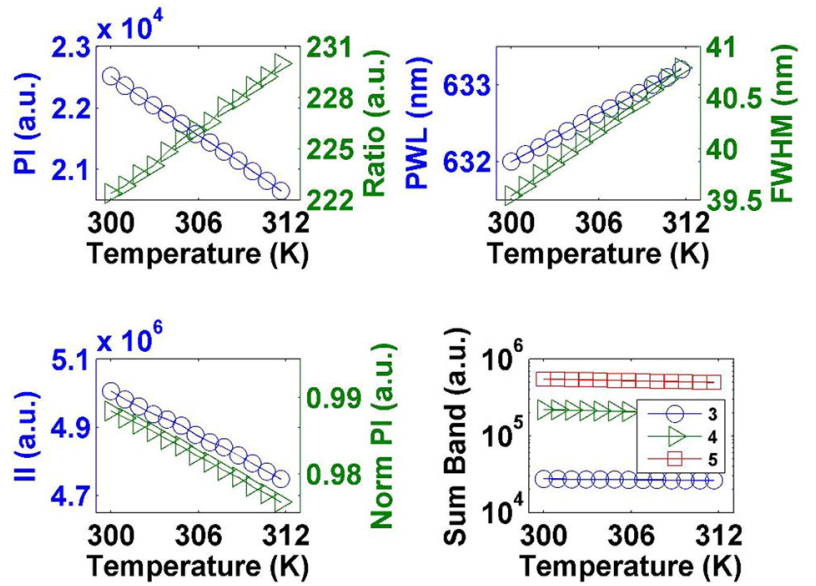

FIG. 8. Linear curve fits of peak intensity (PI), integrated intensity (II), peak wavelength (PWL), ratio, full-width half maximum (FWHM), peaknormalized intensity at a single wavelength (Norm PI), integrated sum bands based on the simulated calibration spectra at known temperatures $(300 \mathrm{~K}-312 \mathrm{~K})$ fit of the ratio. In these cases, the improvement of the fit was not significant $(0.15 / 0.16$ versus 0.19 for the single parameter NN), but the multi-parameter neural networks still provided an order of magnitude improvement. The curve fit with the poorest performance (PI, 40 normalized intensities, $2.84 \mathrm{~K})$ had a neural network with the best performance $(0.29 \mathrm{~K})$, which is an improvement by a factor of 3 compared to the linear fit explicitly used in creating the simulated spectra $(1.02 \mathrm{~K})$.

\section{v. CONCLUSIONS}

$\mathrm{CdSe} / \mathrm{ZnS}$ quantum dots have been shown to be viable fluorescent dyes to be used as a non-contact temperature sensor, with the surface temperature being reconstructed from their fluorescence spectrum by an artificial neural network and improved performance in terms of stability, compared to Rhodamine B, an organic dye. Out of the five neural networks investigated in this work, based on the different spectral shape features, numerical simulations pointed out that the neural network based on the normalized intensity provides the greatest accuracy. The overall RMS uncertainty from simulation of the method to reconstruct the temperature was $0.29 \mathrm{~K}$ by a neural network trained on the peak normalized intensities at 40 wavelengths compared to $1 \mathrm{~K}$ based on reconstruction by only peak intensity, also from simulation. 
This is an improvement compared to an uncertainty of $1.8 \mathrm{~K}$ based on a linear fit of the normalized intensity at a single wavelength and $1.1 \mathrm{~K}$ based on a linear fit, both from experimentally calibrated data. In a comparison to multiple types of curve fits and single parameter input neural networks, the multi-parameter input neural network consistently provided the best performance in temperature reconstruction in both simulation and experiment. This improvement allows for improved thermal characterization of materials and monitoring of thermal processes via a less intrusive temperature measurement.

\section{ACKNOWLEDGMENTS}

The authors would like to thank the members of the USU Multiscale Thermalphysics Lab and the KUL Soft Matter and Biophysics Lab. L. Liu acknowledges the support of the Chinese Scholarship Council (CSC).

${ }^{1}$ X.-D. Wang, O. S. Wolfbeis, and R. J. Meier, Chem. Soc. Rev. 42, 7834 (2013).

${ }^{2}$ Y. He, Ouantum Dots-Based Biological Fluorescent Probes for In Vitro and In Vivo Imaging (INTECH Open Access Publisher, 2012).

${ }^{3}$ E. Betzig, G. H. Patterson, R. Sougrat, O. W. Lindwasser, S. Olenych, J. S. Bonifacino, M. W. Davidson, J. Lippincott-Schwartz, and H. F. Hess, Science 313, 1642 (2006).

${ }^{4}$ J. Lou, T. M. Finegan, P. Mohsen, T. A. Hatton, and P. E. Laibinis, Rev. Anal. Chem. 18, 235 (1999).

${ }^{5}$ K. Okabe, N. Inada, C. Gota, Y. Harada, T. Funatsu, and S. Uchiyama, Nat. Commun. 3, 705 (2012).

${ }^{6}$ T. Glawdel, Z. Almutairi, S. Wang, and C. Ren, Lab Chip 9, 171 (2009).

${ }^{7}$ V. Natrajan and K. Christensen, Meas., Sci. Technol. 20, 015401 (2009).

${ }^{8}$ S. F. Wuister, C. de Mello Donegá, and A. Meijerink, J. Am. Chem. Soc. 126, 10397 (2004).

${ }^{9}$ R. Kariya, H. Maruyama, and F. Arai, in Proceedings of the 2012 International Symposium on Micro-NanoMechatronics and Human Science (MHS) (IEEE, 2012) pp. 215-217.

${ }^{10}$ H. Maruyama, R. Kariya, and F. Arai, Appl. Phys. Lett. 103, 161905 (2013).

${ }^{11}$ D. Aigner, S. M. Borisov, F. J. O. Fernández, J. F. F. Sánchez, R. Saf, and I. Klimant, Talanta 99, 194 (2012).

${ }^{12} \mathrm{M}$. Sauer, J. Hofkens, and J. Enderlein, Handbook of Fluorescence Spectroscopy and Imaging: From Single Molecules to Ensembles (WileyVCH, 2011), p. 1.

${ }^{13}$ R. Liang, R. Tian, W. Shi, Z. Liu, D. Yan, M. Wei, D. G. Evans, and X. Duan, Chem. Commun. 49, 969 (2013).

${ }^{14}$ P. Haro-González, L. Martínez-Maestro, I. Martín, J. García-Solé, and D. Jaque, Small 8, 2652 (2012).
${ }^{15}$ V. Pilla, E. Munin, N. O. Dantas, A. C. Silva, and A. A. Andrade, Quantum Dots-A Variety of New Applications (INTECH, 2012), p. 3.

${ }^{16}$ U. Resch-Genger, M. Grabolle, S. Cavaliere-Jaricot, R. Nitschke, and T. Nann, Nat. Methods 5, 763 (2008).

${ }^{17}$ M. Chu and G. Liu, IEEE Trans. Nanotechnol. 7, 308 (2008).

${ }^{18}$ M. Chu and Y. Sun, Smart Mater. Struct. 16, 2453 (2007).

${ }^{19}$ C. Xing, T. Munro, B. White, H. Ban, C. Copeland, and R. Lewis, Polymer 55, 4226 (2014).

${ }^{20}$ G. Kucsko, P. Maurer, N. Yao, M. Kubo, H. Noh, P. Lo, H. Park, and M. Lukin, Nature 500, 54 (2013).

${ }^{21}$ A. H. Khalid and K. Kontis, Sensors 8, 5673 (2008).

${ }^{22}$ S. Allison and G. Gillies, Rev. Sci. Instrum. 68, 2615 (1997).

${ }^{23}$ H. Li, F. Chen, and H. Hu, Exp. Fluids 56, 1 (2015).

${ }^{24} \mathrm{H}$. Hu and M. M. Koochesfahani, Meas. Sci. Technol. 17, 1269 (2006).

${ }^{25}$ L. Liu, S. Creten, Y. Firdaus, J. J. A. F. Cuautle, M. Kouyaté, M. Van der Auweraer, and C. Glorieux, Appl. Phys. Lett. 104, 031902 (2014).

${ }^{26}$ C. Glorieux, J. S. Antoniow, M. Chirtoc, I. Chirtoc, and J. Thoen, Anal. Sci. 17, s398 (2002)

${ }^{27}$ J. Ravi, Y. Lu, S. Longuemart, S. Paoloni, H. Pfeiffer, J. Thoen, and C. Glorieux, J. Appl. Phys. 97, 014701 (2005).

${ }^{28}$ V. M. Krasnopolsky and H. Schiller, Neural Networks 16, 321 (2003).

${ }^{29}$ S. Chudzik, Meas. Sci. Technol. 23, 065602 (2012).

${ }^{30}$ C. Glorieux, R. L. Voti, J. Thoen, M. Bertolotti, and C. Sibilia, J. Appl. Phys. 85, 7059 (1999).

${ }^{31}$ D. Pugh-Thomas, B. M. Walsh, and M. C. Gupta, Nanotechnology 22, 185503 (2011).

${ }^{32}$ T. Kuriki and T. Miyazaki, Quantum Dots as Global Temperature Measurements (INTECH Open Access Publisher, 2012).

${ }^{33}$ J. Leys, B. Duponchel, S. Longuemart, C. Glorieux, and J. Thoen, Mater. Renewable Sustainable Energy 5, 4 (2016).

${ }^{34}$ F. Zhang, B. Wang, F. Pang, and T. Wang, in Asia Communications and Photonics Conference and Exhibition (International Society for Optics and Photonics, 2010), p. 79900R.

${ }^{35}$ C. G. Copeland, B. E. Bell, C. D. Christensen, and R. V. Lewis, ACS Biomater. Sci. Eng. 1, 577-584 (2015).

${ }^{36}$ J. J. Peterson and T. D. Krauss, Phys. Chem. Chem. Phys. 8, 3851 (2006).

${ }^{37}$ H. Asami, I. Kamiya, and M. Hara, Int. J. Nanosci. 1, 641 (2002).

${ }^{38}$ H. Asami, Y. Abe, T. Ohtsu, I. Kamiya, and M. Hara, J. Phys. Chem. B 107, 12566 (2003).

${ }^{39}$ D. B. Tice, M. T. Frederick, R. P. Chang, and E. A. Weiss, J. Phys. Chem. C 115, 3654 (2011).

${ }^{40}$ J. Bravo, J. Goicoechea, J. M. Corres, F. J. Arregui, and I. R. Matias, in Third European Workshop on Optical Fibre Sensors (International Society for Optics and Photonics, 2007), p. 661919.

${ }^{41}$ S. Ebert, K. Travis, B. Lincoln, and J. Guck, Opt. Express 15, 15493 (2007).

${ }^{42}$ A. Salazar, A. Mendioroz, R. Fuente, and R. Celorrio, J. Appl. Phys. 107, 043508 (2010).

${ }^{43}$ L. Liu, K. Zhong, T. Munro, S. Alvarado, R. Côte, S. Creten, E. Fron, H. Ban, M. Van der Auweraer, N. Roozen et al., J. Appl. Phys. 118, 184906 (2015). 